\title{
Learn from the past and create the future: the 2013 ATS/ERS statement on pulmonary rehabilitation
}

\author{
Sally J. Singh", Richard L. ZuWallack², Chris Garvey ${ }^{3}$ and Martijn A. Spruit ${ }^{4}$, \\ on behalf of the American Thoracic Society/European Respiratory Society task \\ force on pulmonary rehabilitation ${ }^{5}$
}

\begin{abstract}
Affiliations: 'Dept of Respiratory Medicine, Glenfield Hospital, University Hospitals of Leicester NHS Trust, Leicester, UK. ${ }^{2}$ St Francis Hospital Medical Center, Hartford, CT, and ${ }^{3}$ Pulmonary and Cardiac Rehabilitation Dept, Seton Medical Center, Daly City, CA, USA. ${ }^{4}$ Program Development Center, CIRO+ Center of Expertise for Chronic Organ Failure, Horn, The Netherlands. ${ }^{5} \mathrm{~A}$ full list of members of the ATS/ERS task force on pulmonary rehabilitation can be found in the Acknowledgements section.
\end{abstract}

Correspondence: S.J. Singh, Dept of Respiratory Medicine, Glenfield Hospital, University Hospitals of Leicester NHS Trust, Groby Rd, Leicester, LE3 9QP, UK. E-mail: sally.singhवuhl-tr.nhs.uk

0

@ERSpublications

New ATS/ERS statement distils international expertise and experience to guide best practice in pulmonary rehabilitation http://ow.ly/opg60

Pulmonary rehabilitation is an established intervention for the management of chronic obstructive pulmonary disease (COPD); indeed, the delivery of rehabilitation has become routine care for individuals with moderate to severe disease [1]. To guide the delivery of pulmonary rehabilitation, practitioners have looked to the European Respiratory Society (ERS) and the American Thoracic Society (ATS) to offer support in terms of describing the evidence for the intervention and to guide best practice [2]. The new ATS/ERS statement on pulmonary rehabilitation aims to harness international scientific expertise and clinical experience to achieve this challenge [3].

Why a new statement? The 2006 statement provided an excellent reference point for pulmonary rehabilitation [2], but over the past few years there have been several significant developments in the field to warrant a reconsideration of the current position. There is also an obligation to the endorsing societies to ensure that these documents are current and reflect best practice. In June 2010, the process of developing a new statement began. There have been significant developments in our understanding of COPD and the systemic manifestations of the disease, the importance of the intervention in other chronic respiratory disease and the timing of the intervention. The document is complemented by the evidence-based guidelines from the American College of Chest Physicians and American Association of Cardiovascular and Pulmonary Rehabilitation (AACVPR) [4], which formally graded the quality of the scientific evidence, and the AACVPR guidelines for pulmonary rehabilitation programs, which give practical recommendations [5]. By the very nature of a statement, recommendations cannot be made in the 2013 ATS/ERS statement on pulmonary rehabilitation, as these are the preserve of guidelines.

The current ATS/ERS task force was represented by a broad range of professions that are reflected in the interdisciplinary team delivering pulmonary rehabilitation. Compared to the profile of the 2006 authors, the task force co-chairs made the effort to invite a wide ranging team in terms of clinical and scientific background and seniority. The statement has evolved over the past 2 years to the final document presented as a summary document and an online resource with more extensive details [3].

Received: Dec 282012 | Accepted after revision: April 042013

Conflict of interest: None declared.

Copyright @ERS 2013 
The definition of pulmonary rehabilitation has been revised, and that in itself was the cause of some rather significant negotiations, reflecting cultural and operational differences. As a reminder, the previous definition was: "Pulmonary rehabilitation is an evidence-based, multidisciplinary, and comprehensive intervention for patients with chronic respiratory diseases who are symptomatic and often have decreased daily life activities. Integrated into the individualized treatment of the patient, pulmonary rehabilitation is designed to reduce symptoms, optimize functional status, increase participation, and reduce health care costs through stabilizing or reversing systemic manifestations of the disease" [2]. Some aspects of the new definition are aspirational, and to a certain extent reflect the direction of travel for pulmonary rehabilitation over the next few years. The definition does not provide a framework for commissioning a service; this is largely defined by the healthcare systems within which pulmonary rehabilitation is being delivered. The new ATS/ERS definition of pulmonary rehabilitation is:

Pulmonary rehabilitation is a comprehensive intervention based on a thorough patient assessment followed by patient-tailored therapies which include, but are not limited to, exercise training, education and behavior change, designed to improve the physical and psychological condition of people with chronic respiratory disease and to promote the long-term adherence to health-enhancing behaviors.

What are the topics addressed in the revised document? One of the dilemmas discussed at great length was how we define pulmonary rehabilitation and how it is positioned in a package of integrated care [6]. In reality, the precise structure of pulmonary rehabilitation varies across the world [7, 8], although exercise training, education and approaches to support behaviour change are essential. A model identifying where pulmonary rehabilitation is placed in the spectrum of interventions is embedded within the document (fig. 1). It demonstrates clearly that exercise training of course remains the "cornerstone of rehabilitation" (perhaps the most widely quoted phrase from the previous document!), but another important role of pulmonary rehabilitation is to foster competency in collaborative self-management, although this can be an independent activity in its own right. This competency in self-management is facilitated through the entire process of pulmonary rehabilitation and is reflected by a move away from the traditional didactic educational model and move towards an interactive counselling approach to support participants to be actively involved in their disease management.

Tell me and I will forget, show me and I may remember; involve me and I'll understand - Old Chinese proverb.

So, what are the "highlights" of the updated ATS/ERS statement on pulmonary rehabilitation? Fundamentally, the delivery of pulmonary rehabilitation remains a combination of "exercise and education" but the delivery of the intervention has been interrogated over the past few years to develop the pragmatic delivery of pulmonary rehabilitation through to a more scientific exploration of mechanisms.

In the past, exercise training regimes have focused on accurately prescribed regimes; these have been largely cycle-based [9]. However, there is a growing recognition that walking is relatively easy to prescribe, cheap, and a meaningful activity for individuals [10]. As a training modality in its own right, walking can be progressed to a more vigorous form, "Nordic walking" [11]. This appears to be acceptable to patients, provokes a meaningful response compared to a non-exercising control group and has the benefit, like all walking, that it can be continued beyond the period of supervised exercise [11]. Intuitively, it would seem more likely that this type of pragmatic training would support behaviour change, although the impact pulmonary rehabilitation has upon physical activity has yet to be clearly and consistently defined $[12,13]$. However, improving exercise behaviour is a realistic goal and thus has become an important part of the revised definition. The measure of behaviour change with regard to exercise is considered to be reflected in the accumulation of enhanced physical activity during and after the pulmonary rehabilitation period. However, the format of this change may require sophisticated analyses and may not just be a direct measure of steps taken during a given period of time [14]. There have been many detailed interrogations made of the commercially available devices, in order to understand their measurement properties and appropriateness in COPD [14-17]. It would not be difficult to predict that the next revision of the ATS/ERS statement on pulmonary rehabilitation will be heavily populated with this type of research. Interval training has reconfirmed that it is an effective exercise regime that is able to provoke important physiological and clinical changes across a spectrum of disease severity [18-20], but like many other regimes the value of the intervention in improving physical activity is not established and, in particular, requires more sophisticated equipment to deliver.

There has been much interest in the treatment of COPD at the time of an exacerbation. Currently, data is linked to exacerbations of COPD that are managed in hospital. Conventional endurance training may not be practical or indeed feasible during an acute exacerbation; strength training, conversely, provokes a reduced ventilatory load $[21,22]$, and directly addresses the detrimental impact of imposed bed rest upon the skeletal muscle [23]. Studies have explored providing voluntary training and involuntary training at this 
Process

Decision-making

Symptom monitoring Symptom monitoring Medical management Medical management
Enhanced self-efficacy Problem solving

Resource utilisation Collaboration

Emotional management Role management Goal setting

Decision-making Symptom monitoring Medical management
External motivation Social interaction

Enhanced self-efficacy Problem solving

Resource utilisation Collaboration

Emotional management

Role management Goal setting

Decision-making

Symptom monitoring

Medical management
Ongoing support

External motivation

Social interaction

Enhanced self-efficacy

Problem solving

Resource utilisation Collaboration

Emotional management

Role management Goal setting

Decision-making

Symptom monitoring

Medical management

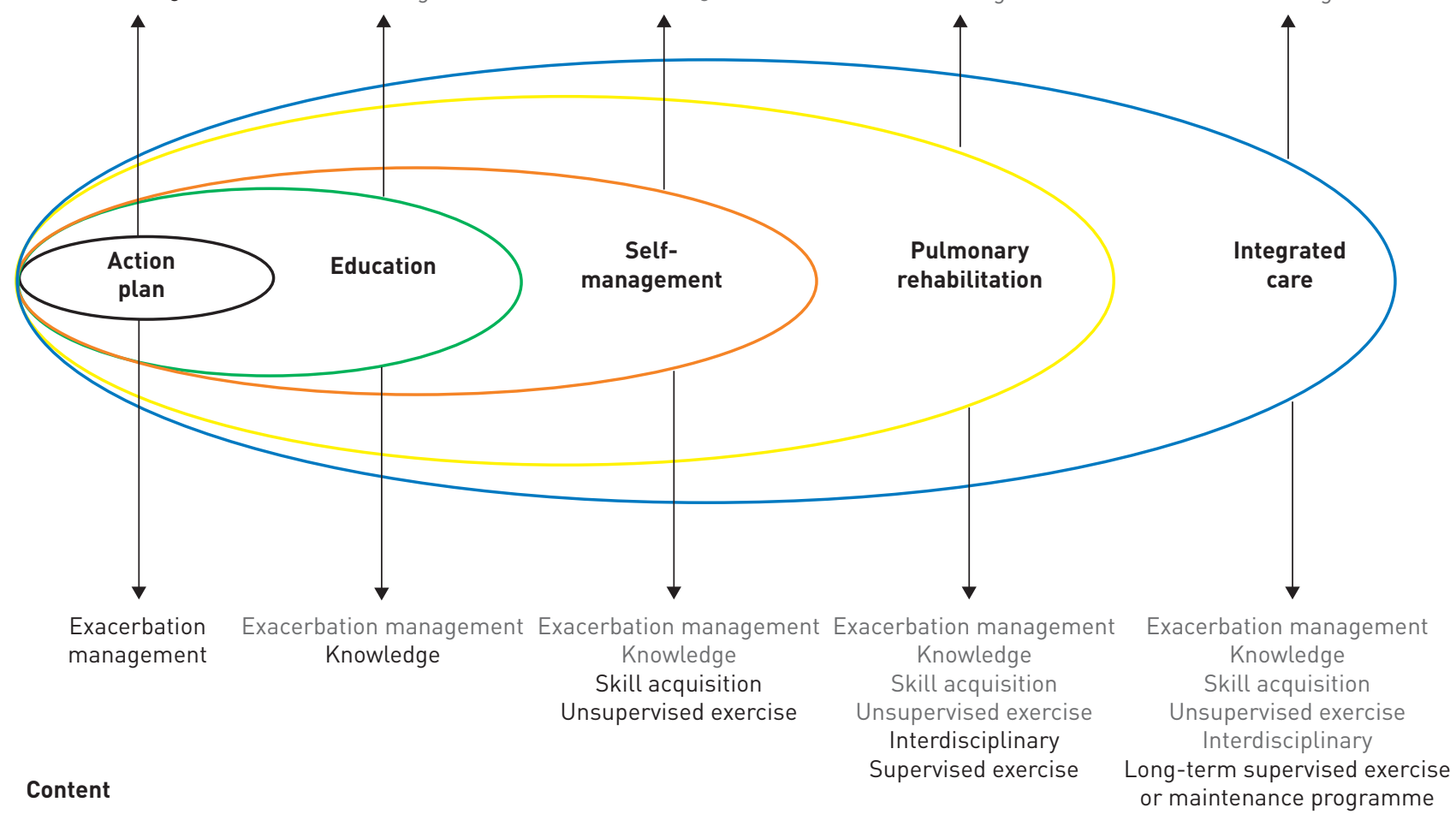

FIGURE 1 Placement of pulmonary rehabilitation in the spectrum of interventions for chronic obstructive pulmonary disease.

time [24-26], and early "proof of concept" data suggest that it is feasible and effective in the short term; further studies are required to understand the broader feasibility and acceptability of these interventions. Post-discharge pulmonary rehabilitation, however, has a very firm evidence base, and a Cochrane Review has conclusively confirmed the clinical value of the intervention [27]. We know it is safe and importantly associated with a reduction in subsequent hospital admissions. One significant issue associated with these trials is the recruitment difficulties that have been described by some authors [28]; there is a need to explore patients' misconceptions about their disease and the value of rehabilitation at this time, to maximise the value of the programme. The importance of the timing has not been well defined, and is an important and outstanding research question.

One of the overriding challenges for pulmonary rehabilitation is access across the globe and, indeed, within any one country provision and uptake is variable. Frequently, the service is not mandated by purchasers or providers. Cardiac rehabilitation is considered obligatory, with an option to opt out rather than opt in. In fact, cardiac rehabilitation has been considered worthy of a World Health Organization definition [29]! It is up to the respiratory community to ensure the evidence is disseminated to all relevant parties and the referral process is endorsed by all senior clinicians. This of course demands close inter-disciplinary working.

The delivery of pulmonary rehabilitation has evolved since the previous statement. Generally, there is an openness to explore different modes of delivery to achieve similar end-points. In many areas, geography 
presents a logistical problem and alternative forms of delivery must be considered. The largest randomised control trial published for several years examined the effectiveness of a home-based exercise training programme. It should be noted that the educational component of the programme was provided by traditional face-to-face sessions, but the exercise was either supervised or unsupervised. An exercise bike was provided for those exercising at home (which, under the current economic climate, may be difficult to achieve). However, what the data clearly demonstrated was that the results of a home exercise training programme were comparable to those achieved in the conventional supervised programme [30]. This challenges conventional wisdom, but it is not the first study to propose that selected patients are able to manage their exercise regime independently, with a carefully prescribed programme and remote supervision $[31,32]$. Remote monitoring can be provided in different ways; we are surrounded by technology but there have been few attempts to capitalise on technology to enhance the rehabilitation experience or diversify in terms of delivery. Mobile phone technology has been successfully employed to improve exercise capacity, compared to a control group [33]. The phones were used to control the speed of walking, monitor progress (via a GPS system) and potentially distract the individual from their perceived breathlessness, as music was played from the phone to aid pacing. These two examples highlight efforts that are being made to increase the scope of pulmonary rehabilitation. There is likely to be increased demand upon pulmonary rehabilitation, as the net will be cast even wider as evidence accumulates to demonstrate comparable benefits in other chronic respiratory disease, most notably interstitial lung disease [34]. The trajectory of benefit and decline appears to be different from the usual COPD population, so we may see an alternative format emerge to maximise the benefit in this group. Tantalising evidence suggests that pulmonary rehabilitation, delivered early in the disease, adds benefit to both the individual and the healthcare system. This should be regarded as a preventative approach (not dissimilar to that in cardiac disease) [35].

In the future, we see the need to increase the applicability and accessibility of pulmonary rehabilitation; to effect behaviour change in order to optimise and maintain outcomes; and to refine this intervention so that it targets the unique needs of the complex patient with a chronic respiratory disease.

\section{Acknowledgements}

The realisation of the 2013 ATS/ERS statement on pulmonary rehabilitation would not have been possible without the support of Miriam Rodriguez (ATS Director Assembly Programs and Program Review Subcommittee), Jessica Wisk (ATS Manager Documents and Ad Hoc Projects), Bridget Nance (ATS Assembly Programs Coordinator), Kevin Wilson (ATS Documents Editor), Judy Corn (Director Documents, Ad Hoc Projects and Patient Education), Prof. Dr Wisia Wedzicha (ERS Guidelines Director), Guy Brusselle (ERS Guidelines Director) and Sandy Sutter (ERS CME and Guidelines Coordinator). Moreover, the task force co-chairs are grateful to the ATS and ERS for funding this statement.

The ATS/ERS task force on pulmonary rehabilitation consisted of: Linda Nici, Providence VA Hospital, Providence, RI, USA; Carolyn Rochester, Yale University School of Medicine, New Haven, CT, USA; Kylie Hill, School of Physiotherapy and Curtin Health Innovation Research Institute, Curtin University, Lung Institute of Western Australia and Centre for Asthma, Allergy and Respiratory Research, University of Western Australia, Perth, Australia; Anne E. Holland, La Trobe University, Alfred Health and Institute for Breathing and Sleep, Melbourne, Australia; Suzanne C. Lareau, College of Nursing, University of Colorado, Denver, CO, USA; William D-C. Man, Respiratory Biomedical Research Unit, Royal Brompton and Harefield NHS Foundation Trust, London, UK; Fabio Pitta, Universidade Estadual de Londrina, Londrina, Brazil; Louise Sewell, Institute for Lung Health, Glenfield Hospital, University Hospitals of Leicester NHS Trust, Leicester, UK; Jonathan Raskin, Beth Israel Medical Center, New York, NY, USA; Jean Bourbeau, Montreal Chest Institute, Montréal, QC, Canada; Rebecca Crouch, Duke University Medical Center, Durham, NC, USA; Frits M.E. Franssen, Program Development Center, CIRO+ center of expertise for chronic organ failure, Horn, the Netherlands; Richard Casaburi, Rehabilitation Clinical Trials Center, Los Angeles Biomedical Research Institute at Harbor-UCLA Medical Center, Torrance, CA, USA; Jan H. Vercoulen, Radboud University Nijmegen Medical Centre, Nijmegen, the Netherlands; Ioannis Vogiatzis, National and Kapodistrian University of Athens, Athens, Greece; Rik Gosselink, Katholieke Universiteit Leuven, University Hospital Leuven, Leuven, Belgium; Enrico M. Clini, University of Modena, Modena, Italy; Tanja W. Effing, Repatriation General Hospital, Adelaide, Australia; Francois Maltais, Institut Universitaire de Cardiologie et de Pneumologie de Québec, Université Laval, Québec, QC, Canada; Job van der Palen, Medisch Spectrum Twente, Enschede, the Netherlands; Thierry Troosters, Katholieke Universiteit Leuven, University Hospital Leuven, Leuven, Belgium; Daisy J.A. Janssen, Program Development Center, CIRO+ center of expertise for chronic organ failure, Horn, the Netherlands; Eileen Collins, Edward Hines, Jr. VA Hospital, Hines, and University of Illinois at Chicago, Chicago, IL USA; Judith Garcia-Aymerich, Centre for Research in Environmental Epidemiology (CREAL), Barcelona, Hospital del Mar Research Institute (IMIM), Barcelona, Universitat Pompeu Fabra, Barcelona, and CIBER de Epidemiologia y Salud Pública (CIBERESP), Spain; Dina Brooks, University of Toronto, Toronto, ON, Canada; Bonnie F. Fahy, Mayo Clinic Hospital, Phoenix, AZ, USA; Milo A. Puhan, Johns Hopkins Bloomberg School of Public Health, Baltimore, MD, USA, and Horten Center for Patient-Oriented Research and Knowledge Transfer, University of Zurich, Zurich, Switzerland; Martine Hoogendoorn, Institute for Medical Technology Assessment/Institute for Healthcare Policy and Management, Erasmus University Rotterdam, Rotterdam, the Netherlands; Rachel Garrod, King's College Hospital NHS Foundation Trust, London, UK; Annemie M.W.J. Schols, NUTRIM School for Nutrition, Toxicology and Metabolism, Maastricht University Medical Center+, Maastricht, the Netherlands; Brian Carlin, Drexel University School of Medicine, Pittsburgh, PA, USA; Roberto Benzo, Mayo Clinic, Rochester, MN, USA; Paula Meek, College of Nursing, University of Colorado, Denver, CO, USA; Mike Morgan, Institute for Lung Health, Glenfield Hospital, University Hospitals of Leicester NHS Trust, Leicester, UK; Maureen P. Rutten-van Mölken, Institute for Medical Technology Assessment/Institute for Healthcare Policy and Management, Erasmus University Rotterdam, 
Rotterdam, the Netherlands; Andrew L. Ries, University of California, San Diego, CA, USA; Barry Make, National Jewish Health, Denver, CO, USA; Roger S. Goldstein, University of Toronto, Toronto, ON, Canada; Claire A. Dowson, University of Otago, Christchurch, New Zealand; Jan L. Brozek, McMaster University, Hamilton, ON, Canada; Claudio F. Donner Mondo Medico, Multidisciplinary and Rehabilitation Outpatient Clinic, Borgomanero, Italy; and Emiel F.M. Wouters, Program Development Center, CIRO+ center of expertise for chronic organ failure, Horn, and NUTRIM School for Nutrition, Toxicology and Metabolism, Maastricht University Medical Center+, Maastricht, the Netherlands.

\section{References}

1 Vestbo J, Hurd SS, Agusti AG, et al. Global strategy for the diagnosis, management and prevention of chronic obstructive pulmonary disease, gold executive summary. Am J Respir Crit Care Med 2013; 187: 347-365.

2 Nici L, Donner C, Wouters E, et al. American Thoracic Society/European Respiratory Society statement on pulmonary rehabilitation. Am J Respir Crit Care Med 2006; 173: 1390-1413.

3 Spruit MA, Singh SJ, Garvey C, et al. An official American Thoracic Society/European Respiratory Society statement: key concepts and advances in pulmonary rehabilitation - an executive summary. Am J Respir Crit Care Med 2013 [In press].

4 Ries AL, Bauldoff GS, Carlin BW, et al. Pulmonary rehabilitation: joint ACCP/AACVPR evidence-based clinical practice guidelines. Chest 2007; 131: 4S-42S.

5 American Association of Cardiovascular and Pulmonary Rehabilitation. Guidelines for Pulmonary Rehabilitation Programs. Champaign, Human Kinetics, 2004.

$6 \quad$ Wagg K. Unravelling self-management for COPD: What next? Chron Respir Dis 2012; 9: 5-7.

7 Brooks D, Sottana R, Bell B, et al. Characterization of pulmonary rehabilitation programs in Canada in 2005. Can Respir J 2007; 14: 87-92.

8 Yohannes A, Stone R, Lowe D, et al. Pulmonary rehabilitation in the United Kingdom. Chron Respir Dis 2011; 8: 193-199.

9 Lacasse Y, Goldstein R, Lasserson TJ, et al. Pulmonary rehabilitation for chronic obstructive pulmonary disease. Cochrane Database Syst Rev 2006; CD003793.

10 Leung RW, Alison JA, McKeough ZJ, et al. Ground walk training improves functional exercise capacity more than cycle training in people with chronic obstructive pulmonary disease (COPD): a randomised trial. J Physiother 2010; 56: $105-112$.

11 Breyer MK, Breyer-Kohansal R, Funk GC, et al. Nordic walking improves daily physical activities in COPD: a randomised controlled trial. Respir Res 2010; 11: 112.

12 Cindy Ng LW, Mackney J, Jenkins S, et al. Does exercise training change physical activity in people with COPD? A systematic review and meta-analysis. Chron Respir Dis 2012; 9: 17-26.

13 Egan C, Deering BM, Blake C, et al. Short term and long term effects of pulmonary rehabilitation on physical activity in COPD. Respir Med 2012; 106: 1671-1679.

14 Pitta F, Troosters T, Probst VS, et al. Quantifying physical activity in daily life with questionnaires and motion sensors in COPD. Eur Respir J 2006; 27: 1040-1055.

15 Annegarn J, Spruit MA, Uszko-Lencer NH, et al. Objective physical activity assessment in patients with chronic organ failure: a validation study of a new single-unit activity monitor. Arch Phys Med Rehabil 2011; 92: 1852-1857.

16 Langer D, Gosselink R, Sena R, et al. Validation of two activity monitors in patients with COPD. Thorax 2009; 64: 641-642.

17 Van Remoortel H, Raste Y, Louvaris Z, et al. Validity of six activity monitors in chronic obstructive pulmonary disease: a comparison with indirect calorimetry. PloS One 2012; 7: e39198.

18 Vogiatzis I, Nanas S, Roussos C. Interval training as an alternative modality to continuous exercise in patients with COPD. Eur Respir J 2002; 20: 12-19.

19 Vogiatzis I, Simoes DC, Stratakos G, et al. Effect of pulmonary rehabilitation on muscle remodelling in cachectic patients with COPD. Eur Respir J 2010; 36: 301-310.

20 Vogiatzis I, Terzis G, Nanas S, et al. Skeletal muscle adaptations to interval training in patients with advanced COPD. Chest 2005; 128: 3838-3845.

21 Sillen MJ, Janssen PP, Akkermans MA, et al. The metabolic response during resistance training and neuromuscular electrical stimulation (NMES) in patients with COPD, a pilot study. Respir Med 2008; 102: 786-789.

22 Probst VS, Troosters T, Pitta F, et al. Cardiopulmonary stress during exercise training in patients with COPD. Eur Respir J 2006; 27: 1110-1118.

23 Spruit MA, Gosselink R, Troosters T, et al. Muscle force during an acute exacerbation in hospitalised patients with COPD and its relationship with CXCL8 and IGF-I. Thorax 2003; 58: 752-756.

24 Abdellaoui A, Prefaut C, Gouzi F, et al. Skeletal muscle effects of electrostimulation after COPD exacerbation: a pilot study. Eur Respir J 2011; 38: 781-788.

25 Giavedoni S, Deans A, McCaughey P, et al. Neuromuscular electrical stimulation prevents muscle function deterioration in exacerbated COPD: a pilot study. Respir Med 2012; 106: 1429-1434.

26 Troosters T, Probst VS, Crul T, et al. Resistance training prevents deterioration in quadriceps muscle function during acute exacerbations of chronic obstructive pulmonary disease. Am J Respir Crit Care Med 2010; 181: 1072-1077.

27 Puhan MA, Gimeno-Santos E, Scharplatz M, et al. Pulmonary rehabilitation following exacerbations of chronic obstructive pulmonary disease. Cochrane Database Syst Rev 2011; CD005305.

28 Puhan MA, Spaar A, Frey M, et al. Early versus late pulmonary rehabilitation in chronic obstructive pulmonary disease patients with acute exacerbations: a randomized trial. Respiration 2012; 83: 499-506.

29 Rehabilitation after cardiovascular disease, with special emphasis on developing countries: report of a WHO committee. World Health Organ Tech Resp Ser 1993; 831: 1-122.

30 Maltais F, Bourbeau J, Shapiro S, et al. Effects of home-based pulmonary rehabilitation in patients with chronic obstructive pulmonary disease: a randomized trial. Ann Intern Med 2008; 149: 869-878.

31 Guell MR, de Lucas P, Galdiz JB, et al. Comparacion de un programa de rehabilitacion domiciliario con uno hospitalario en pacientes con EPOC: estudio multicentrico espanol [Home vs hospital-based pulmonary rehabilitation for patients with chronic obstructive pulmonary disease: a Spanish multicenter trial]. Arch Bronconeumol 2008; 44: 512-518. 
32 Mendes de Oliveira JC, Studart Leitão Filho FS, Malosa Sampaio LM, et al. Outpatient $v s$ home-based pulmonary rehabilitation in COPD: a randomized controlled trial. Multidiscip Respir Med 2010; 5: 401-408.

33 Liu WT, Wang CH, Lin HC, et al. Efficacy of a cell phone-based exercise programme for COPD. Eur Respir J 2008; 32: 651-659.

34 Holland AE, Hill CJ, Conron M, et al. Short term improvement in exercise capacity and symptoms following exercise training in interstitial lung disease. Thorax 2008; 63: 549-554.

35 van Wetering CR, Hoogendoorn M, Mol SJ, et al. Short- and long-term efficacy of a community-based COPD management programme in less advanced COPD: a randomised controlled trial. Thorax 2010; 65: 7-13. 